\title{
GROWTH OF $\mathrm{CdS}_{x} \mathrm{Se}_{1-x}$ NANOPARTICLES IN GLASS MATRIX B Y ISOCHRONAL THERMAL ANNEALING : CONFINED ACOUSTIC PHONONS AND OPTICAL ABSORPTION STUDIES
}

\author{
Anushree Roy and A. K. Sood ${ }^{\star}$ \\ Department of Physics, Indian Institute of Science, \\ Bangalore 560 012, India. \\ *Alsoat Jawaharlal Nehru Centre for Advanced Scientific Research, \\ Jakkur P.O, Jakkur Campus, Bangalore 560 064, India.
}

\begin{abstract}
We have studied the thermal stability of $\mathrm{CdS}_{0.88} \mathrm{Se}_{0.12}$ nanoparticles embedded in a glass matrix as a function of isochronal thermal annealing from $25^{\circ} \mathrm{C}$ to $650^{\circ} \mathrm{C}$. The average size and the size distribution of the particles have been determined from the detailed analysis of optical absorption spectra based on the available results of tight binding calculations and also from the low frequency Raman band which can be attributed to the confined acoustic phonons. It is shown that the particles are stable upto $550{ }^{\circ} \mathrm{C}$ but grow in size after heating at $650{ }^{\circ} \mathrm{C}$.
\end{abstract}

Keywords: nanoparticles, $\mathrm{CdS}_{x} \mathrm{Se}_{1-x}$, optical absorption, confined acoustic phonons.

\section{Introduction}

In recent times the optical properties of semiconductor nanocrystals have attracted considerable attention as they differ significantly from those of the corresponding bulk crystals. From the viewpoint of using them as optical processing devices, semiconductor nanocrystallites embedded in solid matrices such as $\mathrm{NaCl}$ single crystals $[1,2], \mathrm{GeO}_{2}[3,4]$ and $\mathrm{SiO}_{2}$ glasses $[5-10]$ have been investigated extensively. In order to elucidate the optical properties of semiconductor nanocrystals, it is indispensable to consider the zero dimensional confinement effects on the electronic states as well as on the phonons and the electron-phonon interactions. Optical absorption spectroscopy is an important method to obtain information about the electronic states in a system. The blue shifted optical absorption spectrum of the nanocrystallites is a clear signature of quantum size effect in these systems. Recent tight binding calculations give energy levels in good agreement with the measured size dependence of the optical absorption edge of these nanocrystals [11]. Raman scattering is a powerful probe to obtain useful informations on the vibrational states of the particles by investigating confined acoustic phonons, confined optical phonons and surface or interface optical phonon modes. Under resonance conditions, Raman scattering also provides an understanding of electron-phonon interactions.

In this paper, we report the effect of the particle size and size distribution on the optical absorp- tion and vibrational modes in the Schott Filter Glass - GG495 which has $\mathrm{CdS}_{0.88} \mathrm{Se}_{0.12}$ nanocrystals embedded in a borosilicate glass matrix. The objective of the present study was to investigate the stability of the nanoparticles at different temperatures which is a precursor to understand the photodegradation of the filter glasses. This photodegradation in these samples has been already observed by laser irradiation [12]. It has been demonstrated [13] that the Q-switched NdYAG laser induced photodarkening in coloured filter glasses is associated with the growth of $\mathrm{CdS}_{z} \mathrm{Se}_{1-x} \mathrm{mi}$ crocrystallites. The average size and the size distribution of the particles in the samples have been estimated by matching calculated optical absorption spectra with the observed spectra. To calculate the theoretical spectrum, we have assumed the log-normal distribution of particle diameters in conjunction with the allowed electronic transition states as obtained from the effective tight binding model [11]. The average size and the size distribution of the particles have also been estimated from the low frequency Raman (LFR) line [14] which is attributed to the confined acoustic phonons with frequency inversely proportional to the particle diameter. These results are similar to those obtained from the optical absorption measurements. We have studied the stability of these CdSSe particles by isochronal annealing from 25 "C to 650 "C. Orr results show that the particles are almost stable in the matrix till 550 "C. After heating the sample at $650{ }^{\circ} \mathrm{C}$ for $1 / 2 \mathrm{hr}$., the 
average size of the particles increases whereas the sizedistribution remains almost the same.

\section{Experimental}

The optical absorption spectra of the sample GG495 are recorded on $1 \mathrm{~mm}$ thick polished platelets using a Hitachi model 150-20 W-Vis Spectrometer. Raman spectra are recorded at room temperature in $90^{\circ}$ reflection geometry using $5145 \AA$ and $4880 \AA$ lines of the argon ion laser as excitation source (power $\sim 20 \mathrm{~mW}$ ) and computer controlled Spex Ramalog (model 14018) with photon counting electronics. Low frequency Raman spectra are recorded in $\mathbf{W}$ scattering geometry (the electric-fieldvector of the scattered light is parallel to that of the exciting light) as well as in HV geometry (the electric-field vector of the scattered light is perpendicular to that of the exciting light). Isochronal annealings were done by introducing the sample in the temperature controlled furnace maintained at a desired annealing temperature for $1 / 2 \mathrm{hr}$. It is then taken out of the furnace and allowed to cool down to room temperature. The sample was annealed at eight different temperatures from $50^{\circ} \mathrm{C}$ to $550^{\circ} \mathrm{C}$ with a temperature interval of $100^{\circ} \mathrm{C}$ and at $600^{\circ} \mathrm{C}$ and $650^{\circ} \mathrm{C}$. Annealing beyond 700 "C softens the sample and hence experiments were not carried out beyond 650 "C.

\section{Effect of the Particle Size on the Optical Absorption}

Fig. 1(a) and (b) show the optical absorption spec-

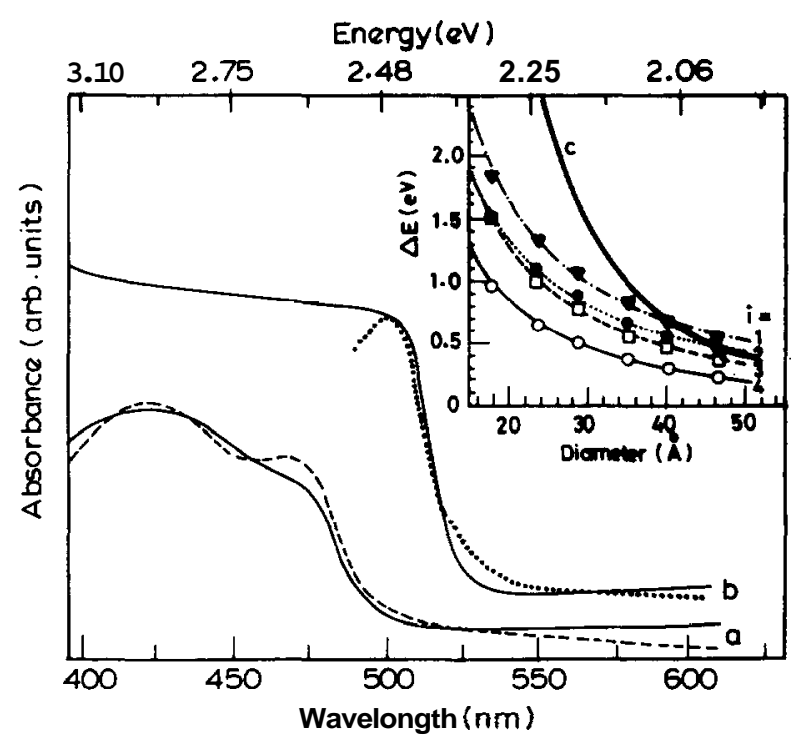

Fig. 1. Optical absorption spectra for the (a) untreated and (b) annealed $\left(650^{\circ} \mathrm{C}\right)$ sample of GG495. The dashed and dotted line shows the calculated spectrum, taking into account log-normal size-distribution. The inset of the figure shows the plot for A E $v s \mathrm{~d}$ for $i$ $=1-4$ (see text). Curve $\mathrm{c}$ in the inset is the plot for $\mathrm{AE}$ $v s \mathrm{~d}$ as estimated by the Effective Mass Approximation model. tra of the untreated and the annealed sample (after annealing the sample at $650^{\circ} \mathrm{C}$ for $1 / 2 \mathrm{hr}$.), respectively. The lowest optical band gap for the bulk $\mathrm{CdS}_{\mathbf{0 . 8 8}} \mathrm{Se}_{, 12}$ crystal $\left(\Omega_{\text {bulk }}\right)$ is $2.35 \mathrm{eV}(528 \mathrm{~nm})$ [15). For the untreated sample, the first absorption band is observed at $470 \mathrm{~nm}(2.64 \mathrm{eV})$ and a broad feature at $425 \mathrm{~nm}(2.92$ $\mathrm{eV}$ ) whereas for the heat-treated sample, the band gap is shifted to $501 \mathrm{~nm}(2.47 \mathrm{eV})$. This blue shift in the $\circ \mathrm{p}$ tical absorption spectra arises from the confinement of the charge carriers in the nanocrystallites. The optical absorption coefficient of a collection of monodispersed nanocrystallites of diameter $\mathrm{d}$ at low temperature is given by

$$
\alpha(\omega) \sim \sum_{i} \frac{f_{i} \Gamma i}{\left(\omega-\Omega_{i}\right)^{2}+\Gamma_{i}^{2}},
$$

where $f_{i}$ is the oscillator strength, $\Omega_{i}$ is the transition frequency and $\Gamma_{i}$ is the half width at half maximum for the ith interband transition. The sum is over all the transitions from filled levels to the unfilled ones. This should results in sharp optical absorption peaks as already observed in $\mathrm{CuCl}$ nanocrystals dispersed in a transparent insulating matrix [16]. However, neither of the above spectra in Fig. 1(a) and (b) shows sharp absorption peaks. This is attributed to the thermal broadening as well as inhomogeneous broadening due to the size distribution. The latter can be included as

$$
\alpha_{\text {observed }}(\omega)=B \sum_{i} \int_{0}^{\infty} d(d) \frac{P(d) f_{i} \Gamma_{i}}{\left[\omega-\Omega_{i}(d)\right]^{2}+\Gamma_{i}^{2}},
$$

where $P(d)$ is the size distribution usually found to be log-normal :

$$
P(d)=\frac{\bar{d}}{d \sqrt{2 \pi} \sigma} \exp \left(-\frac{\ln ^{2}(d / \overline{\mathrm{d}})}{2 \sigma^{2}}\right) .
$$

Here $\overline{\mathrm{d}}$ and $\sigma$ are related to the average size and the size distribution of the particles. The average size $\left(d_{a v}\right)$ of the particles has been estimated numerically by

$$
d_{a v}=\int_{0}^{\infty} d \cdot P(d) d(d)
$$

for a given $\overline{\mathbf{d}}$ and $\sigma$.

To understand the contributions of different electronic states of varying oscillator strengths to the $\mathrm{op}$ tical absorption spectra, we have considered the following transitions as obtained for II-VI semiconductors [11]: $1 \Gamma_{8}^{-}-1 \Gamma_{6}^{+}, 1 \Gamma_{7}^{-}-1 \Gamma_{6}^{+}, 1 \Gamma_{8}^{+}-1 \Gamma_{7}^{-}, 1 \Gamma_{8}^{+}-1 \Gamma_{8}^{-}, 1 \Gamma_{6}^{+}-$ $1 \Gamma_{7}^{-}, 1 \Gamma_{6}^{+}-1 \Gamma_{\mathrm{B}}^{-}, 2 \Gamma_{\mathrm{8}}^{+}-1 \Gamma_{\mathrm{8}}^{-}, 2 \Gamma_{\mathrm{8}}^{+}-1 \Gamma_{7}^{-}, 2 \Gamma_{7}^{+}-1 \Gamma_{8}^{-}, 3 \Gamma_{\mathrm{B}}^{+}-1 \Gamma_{7}^{-}$, $3 \Gamma_{8}^{+}-\mathbf{1} \Gamma_{8}^{-}$. In the effective mass approximation (EMA) model, the shift in transition frequency of the nanocrystallites of diameter $\mathrm{d}$ from the bulk band gap, $\Omega_{\mathrm{bulk}}$, is given by [17]

$$
\Delta E=\frac{2 \hbar^{2} \pi^{2}}{\mu d^{2}}
$$

where $\mu^{-1}=m_{e}^{\star^{-1}}+m_{h}^{\star^{-1}}, m_{e}^{\star}$ and $m_{h}^{\star}$ are the effective masses of the electron and hole, respectively, in bulk crystal $(\mu=0.13$ in electron mass units in the bulk 
Table 1: The variation of the electronic transition frequency with the particle size follows the relation $\mathrm{AE}=$ $\mathrm{A} / \boldsymbol{d}^{x}$, where $\mathrm{AE}=$ bulk band gap -confinement energy+ coulomb energy of the system.

\begin{tabular}{llllll}
\hline$i$ & $\begin{array}{l}\text { electronic } \\
\text { transitions }\end{array}$ & $A$, & $x_{i}$ & $f_{i}$ & $\begin{array}{l}\Gamma_{i} \\
(\mathrm{meV})\end{array}$ \\
\hline 1 & $1 \Gamma_{8}^{-}-1 \Gamma_{6}^{+}$ & 84.5 & 1.54 & 4.13 & 36 \\
& $1 \Gamma_{7}^{-}-1 \Gamma_{6}^{+}$ & 84.5 & 1.54 & 1.73 & $3 \mathrm{C}$ \\
\hline & $1 \Gamma_{8}^{+}-1 \Gamma_{7}^{-}$ & 112.4 & $\mathbf{1 . 4 9}$ & 1.48 & 56 \\
$\mathbf{2}$ & $1 \Gamma_{8}^{+}-1 \Gamma_{8}^{-}$ & 112.4 & $\mathbf{1 . 4 9}$ & 2.28 & 50 \\
& $1 \Gamma_{6}^{+}-1 \Gamma_{7}^{-}$ & 112.4 & $\mathbf{1 . 4 9}$ & 1.41 & $\mathbf{5 0}$ \\
& $1 \Gamma_{6}^{+}-1 \Gamma_{8}^{-}$ & 112.4 & 1.49 & 0.69 & $\mathbf{5 0}$ \\
\hline $\mathbf{3}$ & $\Gamma_{8}^{+}-1 \Gamma_{8}^{-}$ & $\mathbf{6 4 . 6}$ & 1.30 & 1.53 & $\mathbf{6 5}$ \\
& $2 \Gamma_{8}^{+}-1 \Gamma_{7}^{-}$ & $\mathbf{6 4 . 6}$ & 1.30 & 0.32 & $\mathbf{6 5}$ \\
\hline $\mathbf{4}$ & $2 \Gamma_{7}^{+}-1 \Gamma_{8}^{-}$ & $\mathbf{7 8 . 8}$ & $\mathbf{1 . 2 9}$ & $\mathbf{1 . 1 2}$ & 80 \\
& $3 \Gamma_{8}^{+}-1 \Gamma_{7}^{-}$ & $\mathbf{7 8 . 8}$ & 1.29 & 0.56 & 80 \\
& $3 \Gamma_{8}^{+}-1 \Gamma_{8}^{-}$ & $\mathbf{7 8 . 8}$ & $\mathbf{1 . 2 9}$ & 0.23 & 80 \\
\hline
\end{tabular}

crystal). It has been shown [11] that the EMA overestimates the confinement energy A E and the effective bond orbital model within the tight binding approximation leads to a very good agreement with experiments. Inset of Fig. 1 shows the results of the EMA (curve c) for the lowest band gap and the corresponding tight binding results are shown by the curve marked $i$ $=1$. In order to calculate the absorption spectra, we need to know an analytic form for the dependence of transition energies $\Omega_{i}(d)$ on size. This has been obtained by fitting the calculated values (shown by the points in inset of Fig. 1) of $\mathrm{AE}$ to an empirical form $\mathrm{A} \mathrm{E}=A_{i} / d_{i}^{\boldsymbol{x}_{i}}$ (recall $\boldsymbol{x}_{\boldsymbol{i}}=\mathbf{2}$ in EMA model). The lines (i= 1-4) in the inset of Fig. 1 show the fit with parameters given in Table $\mathbf{l}$ ( $\mathrm{AE}$ is expressed in $\mathrm{eV}$ and $d$ is in $\AA$ unit). For a few of the above transitions, corresponding to a particular $\boldsymbol{i}$ in Table 1, the transition energies have the same size dependence. Taking

$$
\Omega_{i}(d)=\Omega_{\text {bulk }}+A_{i} / d^{x_{i}}
$$

in Eq. 2 and using Eq. 3, the dotted line in Fig. 1 shows the $\alpha(\omega)$ calculated with the parameters of Table 1. The best fit is obtained for the size distribution parameters $\sigma=0.2$ and $\overline{\mathrm{d}}=46 \AA$ (which correspond to an average size of the particles of $47 \AA$ and FWHM of $21 \AA$ ) for the untreated sample. However, for the curve $b$, we have to include many more transitions (i >4) for which the size dependence is not known. We have fitted only the absorption edge (as shown in Fig. $\mathbf{1}$ by the dotted line). This procedure gives $\mathbf{d}=\mathbf{6 5} \AA$ and $\sigma=\mathbf{0 . 1 6}$.

\section{Confined Acoustic Phonons}

Low frequency Raman (LFR) peaks, attributed to the confined acoustic phonons, have been observed in spherical nanocrystallites in a nucleated glass; Ge nanocrystals embedded in $\mathrm{GeO}_{2}$ glass [18], Ag nanocrystals in alkali halides [19] and $\mathrm{SiO}_{2}$ glass [20], $\mathrm{CdS}$ nanocrys- tals in $\mathrm{GeO}_{2}$ glass [21] and $\mathrm{CdS}_{\boldsymbol{z}} \mathrm{Se}_{1-x}$ nanocrystals in glass matrix [22-24]. These modes were described, first by Lamb, as the vibrational eigenfunctions of a homogeneous elastic sphere of diameter $d$ with stress-free boundary condition $[14,25]$. In other words, these are eigenfrequencies of the confined acoustic modes with allowed wavevectors equal to $\xi_{l, n}^{S} / d$ and $\eta_{l, n}^{T} / d$ for spheroidal and torsional vibrations, respectively. Here $\xi_{l, n}^{S}$ depends only on the ratio of longitudinal sound velocity $v_{l}$ to the transverse sound velocity $v_{t}$ in the particle and $\eta_{l, n}^{T}$ is independent of material parameters. The modes are characterized by the angular quantum number 1 (for spheroidal modes $l=0,1,2, \ldots$ whereas for torsional modes $1 \geq 1)$ and $\mathbf{n}(=0,1,2, \ldots)$. It has been shown that out of these, the modes with $\mathbf{n}=0$ and 1 $\geq \mathbf{2}$ are the surface modes with larger amplitudes near the surface of the nanocrystals; and the higher modes $(n \geq 1)$ are the inner modes [25]. The solution of the eigenvalue equation for $v_{l} / v_{t}=2.3$ (as for $\mathrm{CdS}_{x} \mathrm{Se}_{1-x} v_{l}$ $=3655 \mathrm{~m} / \mathrm{sec}$ and $v_{t}=1589 \mathrm{~m} / \mathrm{sec}$ ) gives the mode frequencies (expressed in $\mathrm{cm}^{-1}$ ) as [21]

$$
\begin{array}{cc}
\mathbf{n}=\mathbf{O} & \omega_{20}^{S}=0.85 \frac{v_{t}}{d c}, \\
\mathbf{n}=1 & \omega_{01}^{S}=\mathbf{0 . 7 5 2}, \omega_{11}^{S}=1.14 \frac{v_{t}^{*}}{d c}, \omega_{21}^{S}=1.64 \frac{v_{t}^{t}}{d c} \\
n=2 & \omega_{02}^{S}=1.89 \frac{v_{t}}{d c}, \quad \omega_{12}^{S}=2.29 \frac{v_{t}}{d c}, \quad \omega_{22}^{S}=2.74 \frac{v_{t}}{d c}, \\
\mathbf{n}=\mathbf{O} & \omega_{20}^{T}=0.80 \frac{v_{t}}{d c}, \\
\mathrm{n}=1 & \omega_{11}^{T}=1.79 \frac{u_{t}}{d c}, \quad \omega_{21}^{T}=2.29 \frac{v_{t}}{d c}, \\
\mathbf{n}=2 & \omega_{12}^{T}=2.89 \frac{v_{t}}{d c}, \quad \omega_{22}^{T}=3.33 \frac{v_{t}}{d c},
\end{array}
$$

where $c$ is the speed of the light in vacuum $\left(3 \times 10^{10}\right.$ $\mathrm{cm} / \mathrm{sec}$ ). $v_{t}$ and $d$ are in units of $\mathrm{cm} / \mathrm{sec}$ and $\mathrm{cm}$, respectively.

The vibrations must belong to the irreducible re $\mathrm{p}$ resentations of the rotation inversion group $\mathrm{O}(3)$ of the sphere, labelled as $\mathrm{D}_{1}^{g}$ and $\mathrm{D}_{l}^{u}(l=0,1,2,3 \cdots, g$ and $u$ mean even and odd upon inversion) [26]. The spheroidal modes transform as $\mathrm{D}_{0}^{g}, \mathrm{D}_{1}^{u}, \mathrm{D}_{2}^{g}, \ldots$ whereas the torsional modes belong to $D_{1}^{g}, D_{2}^{u}, D_{3}^{g}, \ldots$ The Raman transition operator for dipole allowed scattering (a second rank tensor) belongs to $\mathrm{D}_{0}^{g}$ and $\mathrm{D}_{2}^{g}$ whereas the dipole operator responsible for the infrared absorp tion belongs to $D_{1}^{u}$. Therefore, the only Raman active modes should be from the symmetrical product $\left[\mathrm{D}_{1}^{u} \times \mathrm{D}_{1}^{u}\right]_{\text {sym }}=\mathrm{D}_{0}^{g}+\mathrm{D}_{2}^{g}$, i.e. only the spheroidal modes with $l=0$ or $1=\mathbf{2}$ (quadrupolar mode) are Raman active. This analysis tells that the torsional modes are not Raman active. This is different from the analysis of Fuji et al. [20] and Tanaka et al. [21], based only on the parity of the vibrational wavefunctions, where the spheroidal modes for all even $l$ and the torsional modes of all odd $l$ are Raman active. The spheroidal mode $\mathrm{D}_{0}^{g}$ is completely polarized whereas $\mathrm{D}_{2}^{g}$ are par- 
tially polarized [26]. For spheroidal shaped (ellipsoid of revolution) particles, the $(2 l+1)$ degeneracy of the $\mathrm{D}_{2}^{g}$ mode is lifted into three modes for $\mathrm{m}= \pm 2, \mathrm{~m}= \pm \mathbf{1}$, and $m=0$. The above selection rules are for dipole allowed Raman scattering.

Fig. 2 shows the low frequency Raman spectrum of the sample in polarized (W)and depolarized (HV) configurations obtained using the $5145 \AA$ line of an argon ion laser (power $\sim 20 \mathrm{~mW}$ ). The Raman peak a $\mathrm{p}$ pears at $32 \mathrm{~cm}^{-1}$ in the VV configuration alone. Since only the spheroidal modes with even 1 are Raman active [21], in the inset of Fig. 2 we have plotted $w v s d^{-1}$ (Eq. 7) for spheroidal modes with $\boldsymbol{n}=0,1$ and 2 ; and 1 $=0$ and 2 . The filled circle in the figure corresponds to the LFR peak position at $32 \mathrm{~cm}^{-1}$ and the average particle size $47 \AA$ as obtained from the optical absorption spectrum of the same sample. It suggests that the LFR peak at $32 \mathrm{~cm}^{-1}$ of the untreated sample corresponds to $\omega_{22}^{S}$ mode which gives the average particle diameter to be $45 \AA$. The absence of the spheroidal modes with $l=2$ and $n=0$ can be due to the close contact of the particles with the host matrix which suppresses the surface modes [25]. The spheroidal modes, which do not change the aspect ratio, are expected to be less coupled to the radiation [19]. We also find that the LFR peak is almost absent when recorded with $4880 \AA$ line of argon ion laser, implying resonance enhancement at $5145 \AA$ excitation [27].

Taking into account the instrumental broadening, the line width of an acoustic/optic phonon for a bulk crystal at room temperature is $\sim \mathbf{4} \mathrm{cm}^{-1}$. The broad

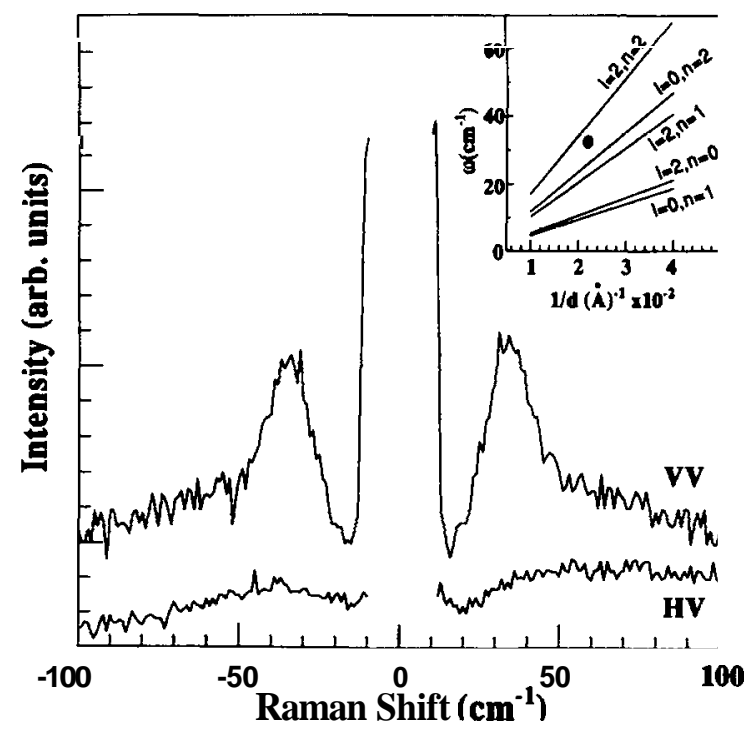

Fig. 2. Stokes and anti-Stokes Raman spectra of the sample GG495 in the polarized (VV) and depolarized (HV) configuration using $5145 \AA$ line of argon ion laser. The inset of the figure shows the $w v s d$ plot for $l=0$ and 2 using Eq. 7 (see text).

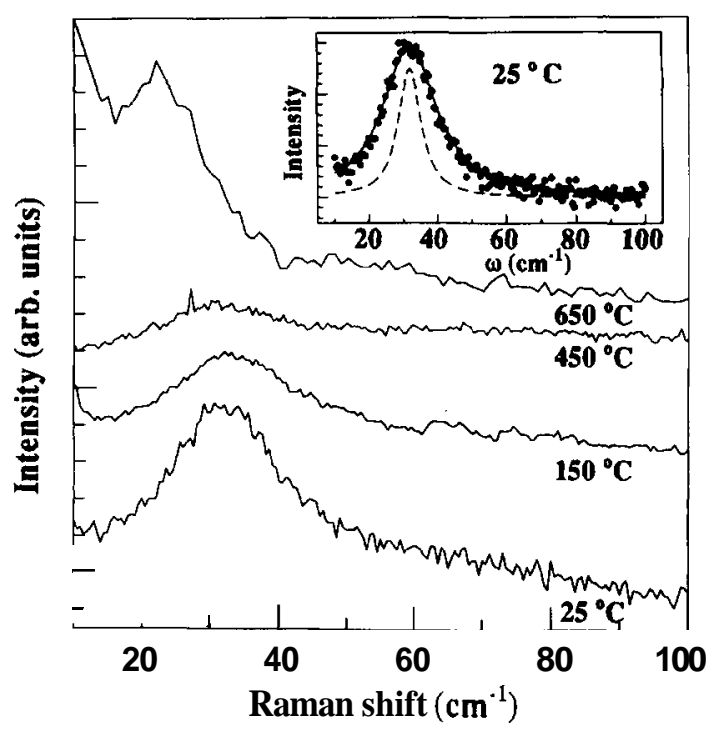

Fig. 3. The low frequency Raman spectra for GG495 at different annealing temperatures. The inset of the figure shows the measured low frequency Raman line (dots) of GG495 at room temperature. Dashed line represents a Lorentzian function corresponding to $\omega_{22}^{S}$ and uniform particles of diameter $45 \AA$. The solid line shows the resultant lineshape using Eq. 3 for $\log$ normal size distribution with $\overline{\mathrm{d}}=45 \AA$ and $\sigma=0.2$.

line width of $17 \mathrm{~cm}^{-1}$ of the low frequency mode in the untreated sample [inset of Fig. 3] should be related to the particle size distribution $P(d)$ :

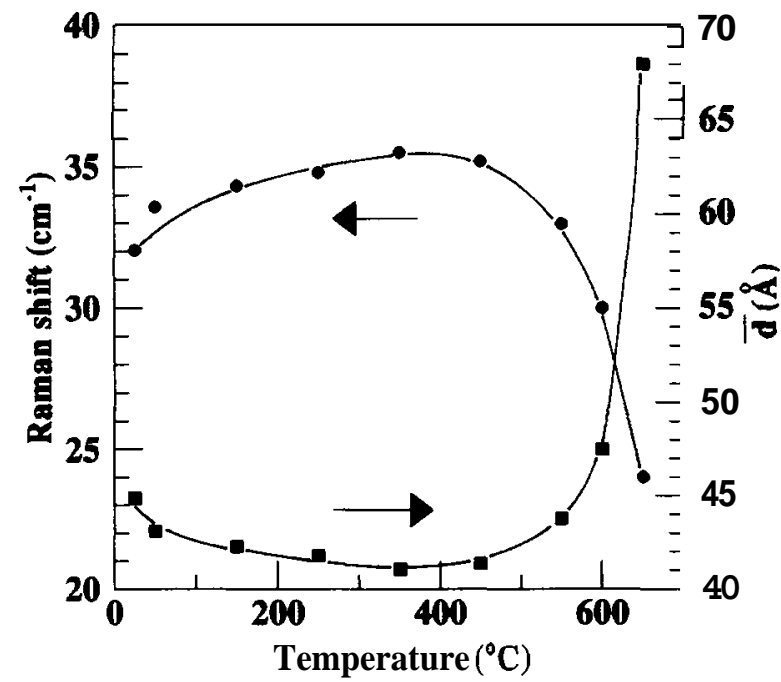

Fig. 4. The variation of the low frequency Raman peak (filled circles) and the average diameter of the particle ( filled squares) with temperature. 
Table 2: Estimation of the size and the size distribution of the $\mathrm{Cd} S_{x} \mathrm{Se}_{1-x}$ of the particles in the sample GG495 as studied by optical absorption spectroscopy (OAS) and low frequency Raman spectroscopy (LFR).

\begin{tabular}{|c|c|c|c|c|c|c|}
\hline \multirow[t]{2}{*}{ Sample } & \multicolumn{3}{|c|}{ OAS measuren ints } & \multicolumn{3}{|c|}{ LFR measurements } \\
\hline & $\begin{array}{c}\text { absorption } \\
\text { peak } \\
(\mathrm{nm})\end{array}$ & $\begin{array}{l}\overline{\mathrm{d}} \\
\AA\end{array}$ & $\sigma$ & $\begin{array}{c}\text { LFR } \\
\text { peak } \\
\left(\mathbf{c m}^{-1}\right)\end{array}$ & $\begin{array}{l}\overline{\mathrm{d}} \\
\AA\end{array}$ & $\boldsymbol{\sigma}$ \\
\hline $\begin{array}{c}\text { GG495 } \\
\text { (untreated) }\end{array}$ & 470 & 46 & 0.2 & 32 & 45 & 0.2 \\
\hline $\begin{array}{c}\text { GG495 } \\
\text { (annealed) }\end{array}$ & 501 & 65 & 0.16 & 24 & 68 & 0.16 \\
\hline
\end{tabular}

$$
I_{L F R}(\omega)=C \int_{0}^{\infty} d(d) \frac{\gamma P(d)}{[\omega-\omega(d)]^{2}+\gamma^{2}},
$$

where $\omega(d)=\omega_{22}^{S}=2.74 \frac{v_{s}}{d c}$ and $\gamma$ is the typical life time plus instrumental broadening of the mode (taken to be $4 \mathrm{~cm}^{-1}$ ). Assuming the particle size distribution to be same as in Eq. 3, the solid line in the inset of Fig. 3 shows the resultant line shape using Eq. 8. Here also the agreement between the calculated lineshape and the data is very good. This analysis yields the values of $\overline{\mathrm{d}}=\mathbf{4 5} \AA$ and $\boldsymbol{\sigma}=\mathbf{0 . 2}$, which correspond to the average size of the particles to be $46 \AA$ and FWHM of $20 \AA$.

To study the stability of the sample with heat treatment, we annealed the sample at different temperatures. The low frequency phonon spectra at four different annealing temperatures are shown in Fig. 3. The low frequency Raman peak at $32 \mathrm{~cm}^{-1}$ almost remains constant till $550{ }^{\circ} \mathrm{C}$. Thermal annealing of the sample at $650{ }^{\circ} \mathrm{C}$ results in a red shift of this low frequency peak to $24 \mathbf{c m}^{-1}$. The filled circles in Fig. 4 show the variation of the LFR peak position and the squares show the deduced variation of $\bar{d}$ with the annealing temperature as obtained by the non-linear least square fitting of the data using Eq. 8. It can be seen that $\bar{d}$ slowly decreases from $45 \AA$ (at room temperature) to $41 \AA$ at $350{ }^{\circ} \mathrm{C}$ annealing. Then it increases to $68 \AA$ at $650{ }^{\circ} \mathrm{C}$. Throughout the temperature range the width of the low frequency Raman peak almost remains constant within the experimental error. The spectrum of the annealed sample (after heating it at $650{ }^{\circ} \mathrm{C}$ for $1 / 2$ hr.) when fitted with Eq. 8 gives $\overline{\mathbf{d}}=\mathbf{6 8} \AA$ and $\boldsymbol{\sigma}=$ $0.16 i$.e. the average size of the particles increases to $68 \AA$ after heating the sample at $650{ }^{\circ} \mathrm{C}$ for $1 / 2 \mathrm{hr}$. In Table 2 we have summarized the above results obtained from the optical absorption spectra and low frequency Raman measurements for the untreated and annealed sample of GG495. We find that the values of $\sigma$ and $\mathbf{d}$ estimated from the two measurements agree very well.

We have also recorded the first order Raman spectra of the sample as a function of annealing temperatures. The peak position remains at $\mathbf{- 3 0 3} \mathbf{c m}^{-1}$. The first order Raman line does not show the red shift expected in a nanoparticle [28) presumably due to the compressive stresses on the particle from the host matrix.

\section{Conclusions}

We have discussed the quantum size effects on electronic excitations as studied by optical absorption spectroscopy in $\mathbf{C d S}_{\mathbf{0 . 8 8}} \mathbf{S e}_{\mathbf{0 . 1 2}}$ particles embedded in a glass matrix. The effect of the size distribution on the o $p$ tical absorption has been taken into account, with inputs from the tight binding model for the transition frequencies. The average size and size distribution of the particles have also been estimated from the low frequency Raman band associated with the confined acoustic phonons. These results have been compared with those obtained from the optical absorption measurements. The stability of the nanoparticles has been studied as a function of isochronal thermal annealing up to 650 "C. We find that the nanoparticles are stable in the matrix till annealing temperatures of $\mathbf{5 5 0}^{\circ} \mathrm{C}$. For further annealing $(\mathbf{6 5 0} " \mathrm{C}$ for $\mathbf{1 / 2} \mathbf{~ h r}$.) the average size of the particles increases by $\mathbf{3 2 \%}$. It would be useful to do similar low frequency Raman measurements on photodegraded filter glasses to understand the laserinduced changes.

Acknowledgement - Authors thank K.C. Rustagi for providing the sample and also for helpful discussions and AKS thanks Department of Science and Technology for financial assistance.

\section{References}

[1] T. Itoh, Y. Iwabuchi and M. Kataoka, Phys. Status Solidi B145, 567 (1988).

[2] T. Itoh, Y. Iwabuchi and T. Kirihara, Phys. Status Solidi B146, 531 (1988).

[3] T. Arai, H. Fujimura, I. Umezu, t. Ogawa and A. Fuji, Jpn. J. Appl. Phys. 28, 484 (1989).

[4) T.Inokuma, T. Arai and M. Ishiwaka, Phys. Rev. B42, 11093 (1990).
[5] A. Nakamura, H. Yamada and T. Tokizaki, phys. Rev. B40, 8585 (1989).

[6] A.I. Ekimov, Al. L. Efros and A.A. Onushenko, Solid State Commun. 56, 921 (1985).

[7] N.F. Borrelli, D.W. Hall, H.G. Holland and D.W. Smith, J. Appl. Phys. 61, 5399 (1987).

[8] S. Hayashi,M. Fuji and K. Yamamoto, Jpn. J. Appl. Phys. 28, L1464 (1989). 
[9] R.K. Jain and R.C. Lind, J. Opt. Soc. America 73, 647 (1983).

[10] J.P. Zheng and H.S. Kwok, Appl. Phys. Lett. 54, 1 (1989).

[11] L.M.Lavanya, and S.V. Nair, Phys. Rev. B 47, 7132 (1993).

[12] P. Roussignol, D. Richard, J. Lukasik and C. Flytzanis, J. Opt. Soc. America B4, 5 (1987).

[13] T. Yanagawa and H. Nakano, Appl. Phys. Lett. 59, 1690 (1991).

[14] H. Lamb, Proc. Lond. Math. 13, 189 (1882).

[15] M. Hayek and O. Brafman, in Light Scattering in Solids, edited by M. Balkanski (Flammirion, Paris), 76 (1971).

[16] A.I. Ekimov and A.A. Onushchenko, Sov. Phys. Semicond. 16775 (1982).

(17) AI. L. Efros and A. L. Efros, Soviet Phys. Semicond. 16, 772 (1982).

[18] N. N. Ovsyuk, E.B. Gorokhov, V.V. Grischenko, and A.P. Shebanin, Sov. Phys. JETP Lett. 47, 298 (1988).
[19] G. Mariotto, M. Montagna, G. Villiani, E. Duval, S. Lefrant, E. Rzepka, and C. Mai, Europhys. Lett. 6, 2391988.

[20] M. Fuji, T. Nagareda, S. Hayashi, and K. Yamamoto, Phys. Rev. B44, 6243 (1991).

[21] A. Tanaka, S. Onari, and T. Arai, Phys. Rev. B47, 1237 (1993).

[22] A.V. Baranov, V.I. Petrov, Ya. B. Bobovich, Opt. Spectroscopy 72, 314 (1992).

[23] B. Champagnon, B. Andrianasolo, E. Duval, J. Chem. Phys. 945237 (1991).

[24] B. Champagnon, B. Andrianasolo, A. Ramos, M. Gandais, M. Allais, and J-P. Benoit, J. Appl. Phys., 73, 2775 (1993).

[25] A. Tamura, K. Higeta and T. Ichinokawa, J. Phys. $C$ : Solid State Phys. 15, 4975 (1982).

[26] E. Duval, Phys. Rev. B46 5795 (1992).

[27] Anushree Roy, Ph.D thesis, Indian Institute of Science, Bangalore, India (unpublished) (1995).

[28] A. Roy, K. Jayaram and A.K. Sood, Solid State Commun. 89, 229 (1994). 